\title{
Note: An Application of the EOQ Model with Nonlinear Holding Cost to Inventory Management of Perishables
}

\author{
Mark Ferguson $^{1} \bullet$ Vaidy Jayaraman ${ }^{2} \bullet$ Gilvan C. Souza $^{3}$ \\ ${ }^{1}$ College of Management, Georgia Inst. of Technology, Atlanta GA 30332; Mark.Ferguson@mgt.gatech.edu \\ ${ }^{2}$ Department of Management, University of Miami, Coral Gables FL 33124; vaidy@miami.edu \\ ${ }^{3}$ Robert H. Smith School of Business, University of Maryland, College Park MD 20742; gsouza@umd.edu
}

\begin{abstract}
In this note, we consider a variation of the economic order quantity (EOQ) model where cumulative holding cost is a nonlinear function of time. This problem has been studied by Weiss (1982), and we here show how it is an approximation of the optimal order quantity for perishable goods, such as milk, and produce, sold in small to medium size grocery stores where there are delivery surcharges due to infrequent ordering, and managers frequently utilize markdowns to stabilize demand as the product's expiration date nears. We show how the holding cost curve parameters can be estimated via a regression approach from the product's usual holding cost (storage plus capital costs), lifetime, and markdown policy. We show in a numerical study that the model provides significant improvement in cost vis-à-vis the classic EOQ model, with a median improvement of $40 \%$. This improvement is more significant for higher daily demand rate, lower holding cost, shorter lifetime, and a markdown policy with steeper discounts.
\end{abstract}

Keywords: Inventory, EOQ model, retailing, perishable products

\section{Introduction}

In this note, we consider an application of the model by Weiss (1982) to an inventory management of perishables problem. The model by Weiss (1982) is a variation of the economic order quantity (EOQ) model where cumulative holding cost is a convex function of time; this is in contrast with the classic EOQ model where holding cost is a linear function of time. More specifically, the cumulative holding cost for one unit that has been stored during $t$ units of time is $H(t)=\tilde{h} t^{\gamma}$, where $\tilde{h}$ and $\gamma \geq 1$ are constants; if $\gamma=1$ then the problem reduces to the classic EOQ model with $\tilde{h}$ being the cost to hold one unit for one time period.

This problem is an approximation of the optimal order quantity for perishable goods, such as milk and produce, sold in small to medium size grocery stores such as WholeFoods. 
Because of their relatively small size, these stores do not receive daily deliveries and often face a fixed delivery charge between $\$ 10$ and $\$ 100$ per delivery; in many cases, there are separate deliveries for each product type. (In contrast, this fee is often waived for large retailers such as Kroger and Wal-Mart, where deliveries may occur five to six times a week.) Product demand and cost are fairly constant over time, however, the cost to stock the product increases over time, as we discuss below.

Because products are perishable, meeting a constant demand over time with an aging product may require markdowns in their prices or removal of spoiled product. The use of either practice can be modeled as convex holding costs with time, as we show through two examples in §3. Our experience with several retailers indicates that retailers usually limit the number of markdowns to two for each batch. The first markdown occurs at roughly half the product's sellable lifetime and is typically $10-50 \%$ of the product's original price. The second markdown occurs at $75 \%$ of product's sellable lifetime and is typically $25-75 \%$ of the original price. For example, a $\$ 4$ gallon of milk with an expected lifetime of 12 days can be marked down to $\$ 2$ at day 6 days and to $\$ 1$ at day 9 . We note that not all retailers practice markdown pricing while others practice it to a lesser extent; other alternatives include selling the older product to restaurants or donating the aged product to food banks.

A second contributor to convex holding cost is spoilage, or variable expected shelf life. Within a product category, the percentage of individual units that spoil each day increases as the product ages. As we show in §3.2, spoilage can be approximated by a convex holding cost curve even in the absence of markdown pricing. In that case, however, the order quantity in the model must be adjusted upwards to account for spoilage.

Variations on the EOQ model are numerous; for a review see Zipkin (2000). Specific reviews of perishable inventory models are given by Raafat (1991) and Nahmias (1982). 
Most papers on perishable inventory models with deterministic demand consider that inventory spoils (decays) with time, at different patterns, and that demand depends on the level of inventory (e.g., Ghare and Scharder 1963; Elsayed and Teresi 1983; Padmanabhan and Vrat 1995).

Deterministic demand inventory models with nonlinear holding costs have been analyzed by, besides Weiss (1982), Goh (1992) and Giri and Chaudhuri (1998). In both Goh (1992) and Giri and Chauduri (1998), however, demand depends on stock level; our application assumes a constant demand rate due to a markdown policy.

Thus, the contribution of this paper is to provide a real-life application of the model by Weiss (1982) to the case of perishable products. We provide a simple methodology to estimate the holding cost curve parameters given a product's lifetime, regular holding (storage + cost of capital) cost, and markdown policy or spoilage curve. We also provide a comprehensive numerical analysis of the benefits of using this policy vis-à-vis the EOQ model, using data provided to us by actual stores.

This note is organized as follows. In §2, we briefly review the model by Weiss. In $\S 3$, we show how one can estimate the holding cost parameters with two real life examples. In $\S 4$, we present a numerical study that demonstrates the value of our model vis-à-vis a regular EOQ policy for inventory management. Finally, we conclude in §5.

\section{Model}

In this section, we quickly review the model by Weiss (1982). Consider a product facing a constant demand rate $d$. Fixed ordering cost is $K$, replenishment lead-time is constant, and holding cost per unit increases with the time $t$ that the product has been in stock according to $H(t)=\tilde{h} t^{\gamma}$, where $\tilde{h}$ and $\gamma \geq 1$ are constants. The firm's objective is to choose 
an order quantity that minimizes average combined ordering and holding costs over an infinite horizon. With an order quantity of $Q$, and constant demand rate $d$, the length of an order cycle is $Q / d$. Because all order cycles are equal, consider the first order cycle $[0, Q / d]$. First, note that $\tilde{h} t^{\gamma}=\int_{0}^{t} \gamma h v^{\gamma-1} d v$; this is the total holding cost if one unit is kept in stock during the period $[0, t]$. During the first cycle, the inventory level varies with time $I(t)=Q-d t$. Thus, the average holding cost during the cycle $[0, Q / d]$ is:

$$
\bar{H}=\frac{1}{Q / d} \int_{0}^{Q} I(t) \gamma \tilde{h} u^{\gamma-1} d u=\frac{1}{Q / d} \int_{0}^{Q}(Q-d u) \gamma \tilde{h} u^{\gamma-1} d u=\frac{\tilde{h} Q^{\gamma}}{(\gamma+1) d^{\gamma-1}} .
$$

Thus, (1) is also the average holding cost in an infinite horizon. Average inventory cost (holding + ordering) is then

$$
F(Q)=\frac{\tilde{h} Q^{\gamma}}{(\gamma+1) d^{\gamma-1}}+\frac{d}{Q} K .
$$

It is easy to verify that (2) is convex in $Q$. Applying the first order condition to the average cost function (2), we obtain the optimal order quantity $Q^{*}$ as

$$
Q^{*}=\sqrt[(\gamma+1)]{\left(1+\frac{1}{\gamma}\right) \frac{K d^{\gamma}}{\tilde{h}}}
$$

Equation (3) agrees with the classical EOQ model when $\gamma=1$.

\section{Estimating Holding Cost Parameters}

In this section, we give two examples that show how the parameters $\tilde{h}$ and $\gamma$ can be estimated, using linear least squares regression, from the product holding cost $h$, the product's lifetime $T$, and a given markdown policy or spoilage curve. Both examples are based on numbers provided to us by a national U.S. grocery chain.

\subsection{Example 1: Markdown Policy}

Consider a two-gallon jug of milk, with $T=12$ days (expiration date), $h=0.01 /$ day, and a markdown policy that decreases the product's price by $\$ 0.50$ on days 5 and 10 . The 
cumulative holding cost curve per unit $H(t)$ is given as a function of time in Table 1. Notice that at day 5, the cumulative holding cost jumps from $\$ 0.04$ to $\$ 0.55(\$ 0.01+\$ 0.50)$, which is a result to the product being marked down; similarly at day 10 .

We use the convex approximation $H(t)=\tilde{h} t^{\gamma}$ to the data in Table 1. Taking logs on both sides yields

$$
\log H(t)=\log \tilde{h}+\gamma \log t .
$$

Table 1: Cumulative holding cost curve for a two-gallon jug of milk

\begin{tabular}{cc}
\hline Day & $\begin{array}{c}\text { Cumulative } \\
\text { Holding Cost } H(t)\end{array}$ \\
\hline 1 & 0.01 \\
2 & 0.02 \\
3 & 0.03 \\
4 & 0.04 \\
5 & 0.55 \\
6 & 0.56 \\
7 & 0.57 \\
8 & 0.58 \\
9 & 0.59 \\
10 & 1.10 \\
11 & 1.11 \\
12 & 1.12 \\
\hline
\end{tabular}

Using a linear regression where the independent variable is $t$, and the dependent variable is $H(t)$, we estimate $\tilde{h}=\exp ($ intercept), and $\gamma=$ slope. For the data in Table 1 , intercept $=-5.16$, slope $=2.21, \mathrm{R}^{2}=0.883$; thus $\tilde{h}=e^{-5.16}=0.0057$, and $\gamma=2.21$. Figure 1 plots the cumulative holding cost and its convex approximation curve $H(t)$. 
Figure 1: Convex approximation for cumulative holding cost for example 1

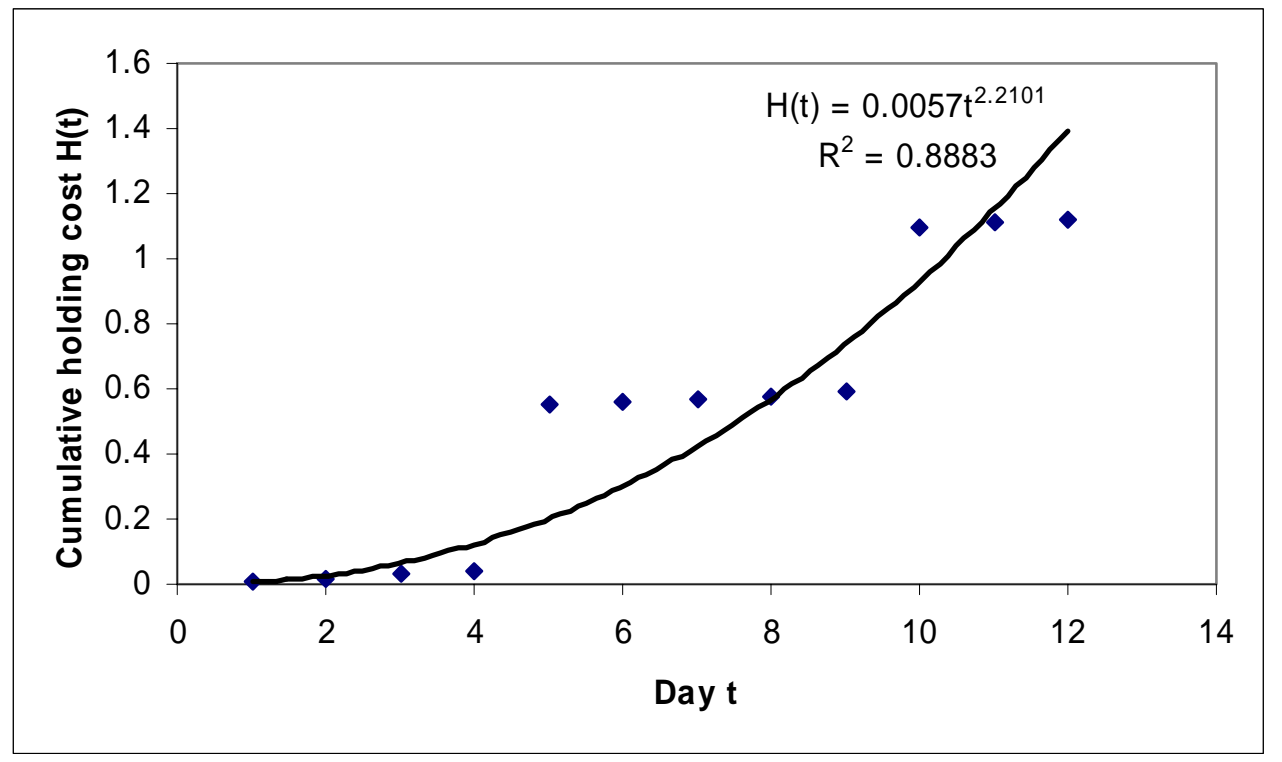

\subsection{Example 2: Spoilage}

Consider a 5 oz. pack of blueberries, with $T=5$ days (expected lifetime), $h=$ $0.01 /$ day, and a cost of $\$ 2$ /unit. Average spoilage, based on historical observations, is 5\%, $7.5 \%, 10 \%$, and $22.5 \%$ of the remaining stock of blueberry packs after the second, third, fourth and fifth days respectively. Thus, cumulative spoilage cost per unit is $(0.05) \$ 2=$ $\$ 0.10$ for the second day; $(0.05+0.075) \$ 2=\$ 0.25$ for the third day, and so forth. Adding the regular storage cost of $\$ 0.01$ per unit per day, we obtain the cumulative holding cost, shown in Table 2. Notice that at day 6, all blackberries are spoiled.

Again, running a linear regression of $\log (H(t))$ vs. $\log (t)$, we obtain intercept $=-$ 4.22, slope $=2.81, \mathrm{R}^{2}=0.987$; thus $\tilde{h}=e^{-5.16}=0.012$, and $\gamma=2.81$. Figure 2 plots cumulative holding cost and its convex approximation curve $H(t)$. 
Table 2: Cumulative holding cost for blueberry pack example

\begin{tabular}{|c|c|c|c|c|c|c|}
\hline Day $t$ & \% Spoilage & \multicolumn{2}{|c|}{$\begin{array}{c}\text { Cumulative } \\
\text { Spoilage Cost }\end{array}$} & $\begin{array}{c}\text { Cumulative } \\
\text { Storage Cost }\end{array}$ & $\begin{array}{r}\text { Cum } \\
\text { Holding }\end{array}$ & $\begin{array}{l}\text { ulative } \\
\text { Cost } H(t)\end{array}$ \\
\hline 1 & $0.0 \%$ & \$ & & 0.01 & $\$$ & 0.01 \\
\hline 2 & $5.0 \%$ & $\$$ & 0.10 & 0.02 & $\$$ & 0.12 \\
\hline 3 & $7.5 \%$ & $\$$ & 0.25 & 0.03 & $\$$ & 0.28 \\
\hline 4 & $10.0 \%$ & $\$$ & 0.45 & 0.04 & $\$$ & 0.49 \\
\hline 5 & $22.5 \%$ & $\$$ & 0.90 & 0.05 & $\$$ & 0.95 \\
\hline 6 & $55.0 \%$ & $\$$ & 2.00 & 0.06 & $\$$ & 2.06 \\
\hline
\end{tabular}

Figure 2: Convex approximation for cumulative holding cost for example 2

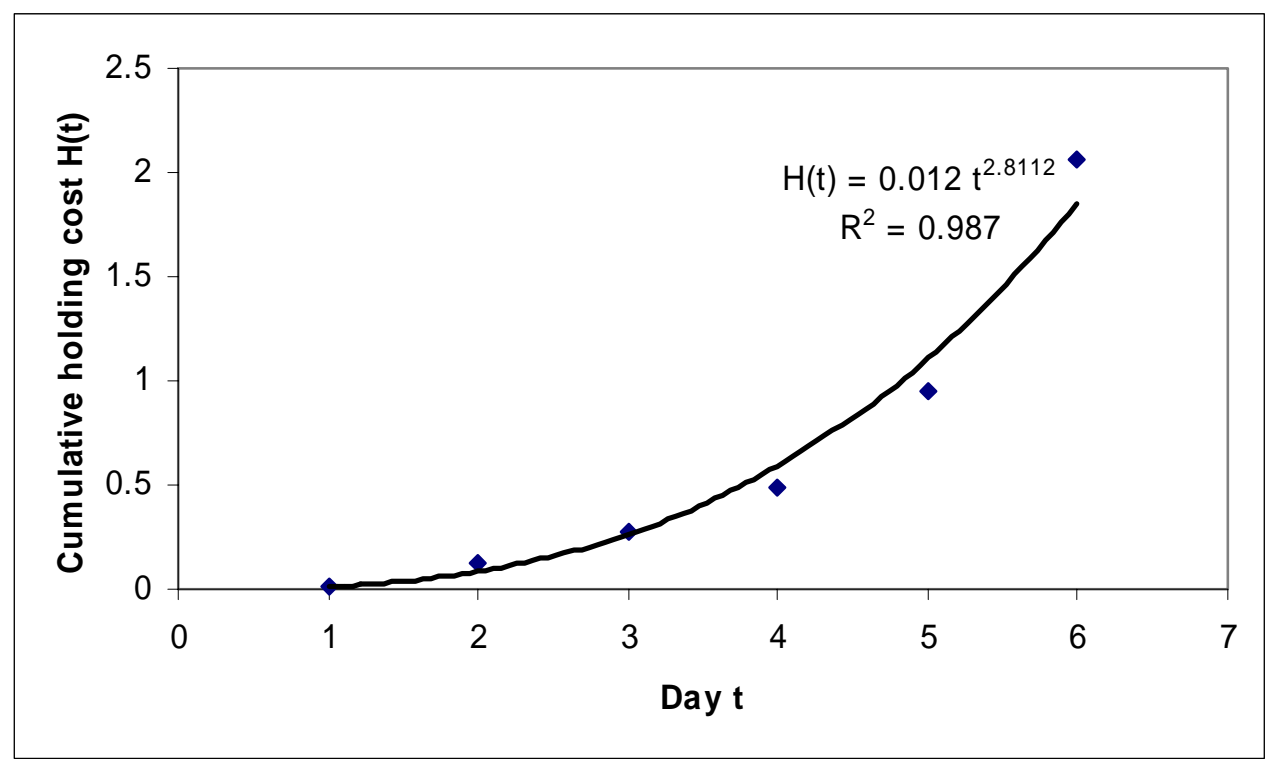

We note that in the case of spoilage, the order quantity should be adjusted upwards to account for the units lost to spoilage, as the model assumes that all units ordered will be used to meet demand. Since the cost of storage is already accounted for by the increasing holding cost term, the final order quantity is the EOQ determined through (3) plus the cumulative number spoiled until the next replenishment. Using the data from the blueberry example above with the fact that $d=40$ packs a day and $K$ is $\$ 30$, the optimal order quantity calculated from (3) is 128 , translating to an order every 3.2 days. Rounding to the nearest integer gives an order ever 3 days or an order quantity of 120 . Adding the spoilage 
adjustment of 7 additional units $(.05 * 80+.075 * 40)$ gives a final order quantity of 127 units every three days.

\section{Numerical Study}

In this section, we present a numerical study that compares how a retailer who uses the model fares against an identical retailer who uses the classic EOQ model for her replenishment decisions. We base our numbers on conversations with produce managers from two national grocery retail chains. We fix two parameters in our analysis: a product that has a unit cost of $\$ 4$, and a fixed delivery charge $K=\$ 50$. We consider various scenarios of the demand rate $d$, holding cost $h$, markdown policy, and product lifetime $T$, as shown in Table 3, which we justify as follows.

We choose five values of $d$, from 10 to 90 , to represent a range of products from low to high daily demand. We consider five values of $h$, from 0.01 to 0.09 , to represent various levels of holding cost per day (storage cost plus cost of capital). We consider two values of T, 6 and 12, to represent perishable products with short and long lifetimes, respectively. Finally, we consider five types of markdown policies, according to the discount given at $1 / 2$ and $3 / 4$ of the product's lifetime, from low discount (discounts of $12.5 \%$ and $25 \%$ of the original product's price, respectively) to high discount (discounts of $50 \%$ and $75 \%$ of the original product's price, respectively).

Table 3: Experimental design used in numerical study

\begin{tabular}{|c|c|}
\hline Parameter & Values \\
\hline$d$ & $10,30,50,70,90$ \\
\hline$H$ & $0.01,0.03,0.05,0.07,0.09$ \\
\hline$T$ & 6,12 \\
\hline $\begin{array}{l}\text { Markdown Policy } \\
\text { type-( } 1 / 2 \text {-life discount, } \\
\text { 3/4-life discount) }\end{array}$ & $\begin{array}{c}\text { 1-(12.5\%, 25\%); 2-(12.5\%, 50\%); 3-(25\%, 50\%), } \\
\text { 4-(25\%, 75\%); 5-(50\%, 75\%) }\end{array}$ \\
\hline
\end{tabular}


Each combination $h, T$, and markdown policy generates a pair of values for $\tilde{h}$ and $\gamma$, which are computed through linear regression, according to the procedure described in $§ 3.1$. The resulting values of $h$ range between 0.036 and 0.081 ; the resulting values of $\gamma$ range between 1.38 and 3.73; finally, the resulting values of $\mathrm{R}^{2}$ range between $83 \%$ and $97 \%$, averaging 91\%. Thus, our convex approximation provides a reasonable fit to the data in all cases.

In total, there are $5^{3} \cdot 2=250$ experimental cells. For each cell, we compute $Q^{*}$ according to (3), and the corresponding classical EOQ solution $Q^{1}=\sqrt{2 d K / h}$. In some cells, the EOQ solution produces a length of order cycle $Q^{1} / d$ greater than $T$; in that case the order quantity is the demand during the product's lifetime. (We do not considering spoilage here). More precisely,

$$
Q^{E O Q}= \begin{cases}\sqrt{2 d K / h}, & \text { if }(\sqrt{2 d K / h} / d)>T \\ T d, & \text { otherwise. }\end{cases}
$$

(We note that in all our experimental design, $Q^{*} / d \leq T$, so such adjustment is not necessary for the optimal solution $Q^{*}$ ). Our performance measure of interest is the deterioration in average cost $F(\cdot)$ of using the EOQ order quantity (5) versus the optimal policy $Q^{*}$, that is

$$
\Delta=100 \% \frac{F\left(Q^{E O Q}\right)-F\left(Q^{*}\right)}{F\left(Q^{*}\right)} .
$$

We report here the main results of our study; our complete results are available upon request. Table 4 provides summary statistics for $\Delta$, and the difference in order cycles (in days) between the EOQ model and the optimal policy, that is, $Q^{E O Q} / d-Q^{*} / d$. We note that $\Delta$ ranges between $1 \%$ and $401 \%$, with a median value of $40 \%$. The difference in order cycles range between 0.4 and 7.6 days, with a median value of 2.5 days, where we note that in $44 \%$ of cells we have $Q^{E O Q}=T d$, that is, the EOQ value $\sqrt{2 d K / h}$ had to be adjusted to the demand during the product's lifetime $T$. This indicates that managers who use the EOQ 
model for perishables are likely to use some heuristic for adjusting the order size, such that the length of the order cycle does not exceed the product's lifetime. Overall, these results point to the significant value of our model vis-à-vis the EOQ model, both in terms of average inventory cost as well as in shorter order cycles, which result in a lower utilization of markdowns, and, consequently a more stable price.

Table 4: Summary statistics for $\Delta$ and difference in order cycles for experimental design

\begin{tabular}{ccc}
\hline Statistic & Value & $\begin{array}{c}\text { Difference in order } \\
\text { cycles (days) }\end{array}$ \\
\hline Average & $69 \%$ & 2.8 \\
Min & $1 \%$ & 0.4 \\
$25^{\text {th }}$ percentile & $16 \%$ & 1.6 \\
Median & $40 \%$ & 2.5 \\
$75^{\text {th }}$ percentile & $90 \%$ & 3.3 \\
Max & $401 \%$ & 7.6 \\
\hline
\end{tabular}

To gain insights into which factors contribute the most to $\Delta$, we average, for each value of a given factor (e.g., $d=10$ ), the values of $\Delta$ across the experimental design; the resulting value is the average value of $\Delta$ when $d=10$ in our experimental design. The results are shown in Table 5. We note that $\Delta$ increases as $d$ increases, $h$ decreases, $T$ decreases, and the discount values increases. A higher value of $d$ indicates a higher scale of operations, and thus the benefit of using the optimal policy is higher. A higher value of $h$ results in shorter order cycles by the EOQ model, which more closely matches the optimal solution. To illustrate, as $h$ increases from 0.01 to 0.03 to 0.05 , the average order cycle for EOQ model $Q^{E O Q} / d$ decreases from 8.8 to 7.4 to 6.6 ; for the optimal policy these order cycles are 4.6, 4.2 and 3.9, respectively. A lower lifetime $T$ indicates a rapidly perishable product, which results in a steeper cumulative holding cost curve (higher $\gamma$ ); consequently a higher value of $\Delta$. To illustrate, when $T=6$ the average value of $\gamma$ is 2.6 , whereas when $T=12$ the average value of $\gamma$ is 2.0. Finally, a steeper markdown policy also results in a steeper cumulative 
holding cost curve, and consequently a higher value of $\Delta$. To illustrate, the average values of $\gamma$ are 1.9 and 2.5 for markdown policies 1 and 5, respectively.

Table 5: Average values of $\Delta$ in experimental design for each factor level

\begin{tabular}{crr}
\hline \multicolumn{2}{c}{ Parameter } & \multicolumn{2}{c}{ Average Value of $\Delta$} \\
\hline $\boldsymbol{d}$ & & \\
& 10 & $22 \%$ \\
& 30 & $61 \%$ \\
& 50 & $80 \%$ \\
& 70 & $91 \%$ \\
$\boldsymbol{h}$ & 90 & $91 \%$ \\
& & \\
& 0.01 & $149 \%$ \\
& 0.03 & $83 \%$ \\
& 0.05 & $52 \%$ \\
& 0.07 & $35 \%$ \\
& 0.09 & $25 \%$ \\
T & & \\
& 6 & $85 \%$ \\
Markdown Policy & 12 & $52 \%$ \\
& 1 & \\
& 2 & $29 \%$ \\
& 3 & $50 \%$ \\
& 4 & $66 \%$ \\
& 5 & $87 \%$ \\
& & $112 \%$ \\
\hline
\end{tabular}

\section{Conclusion}

In this note, we apply the extension of the EOQ model for nonlinear holding cost by Weiss (1982) to the inventory management of perishables in small to medium-sized grocery stores, where managers frequently utilize markdowns to stabilize demand as the product's expiration date nears. We show how the model's holding cost parameters can be estimated via a regression approach from the product’s holding cost, lifetime, and markdown policy.

We show in a numerical study that the model provides significant improvement in cost vis-à-vis the classic EOQ model, with a median improvement of 40\%, ranging between 
$1 \%$ and $401 \%$. This improvement is more significant for higher daily demand rate, lower holding cost, shorter lifetime, and a markdown policy with steeper discounts; in many cases these conditions are associated with a steeper curve of cumulative holding cost vs. time.

\section{References}

Elsayed, E. A., and C. Teresi (1983). “Analysis of Inventory Systems with Deteriorating Items.” International Journal of Production Research, 21(4): 449-460.

Ghare, P. M., and G. P. Scharder (1963). “A Model for Exponentially Decaying Inventory.” Journal of Industrial Engineering 14(5): 238-243.

Giri, B. C., and K. S. Chauduri (1998). “Deterministic Models of Perishable Inventory with Stock-Dependent Demand Rate and Nonlinear Holding Cost.” European Journal of Operational Research 105: 467-474.

Goh, M. (1994). “EOQ Models with General Demand and Holding Cost Functions.” European Journal of Operational Research 73: 50-54.

Nahmias, S. (1982). “Perishable Inventory Theory: A Review.” Operations Research 30(4): 680-708.

Padmanabhan, G., and P. Vrat (1995). “EOQ Models for Perishable Items under Stock Dependent Selling Rate.” European Journal of Operational Research 86: 281-292.

Raafat, F. (1991). "Survey of Literature on Continuously Deteriorating Inventory Models.” Journal of the Operational Research Society 42(1): 27-37.

Weiss, H. (1982). “Economic Order Quantity Models with Nonlinear Holding Costs.” European Journal of Operational Research 9:56-60.

Zipkin, P. (2000). Foundations of Inventory Management. McGrawHill. 